\title{
Effect of ischaemic compression versus kinesiotape on patellar tendinitis
}

\author{
DOI: https://doi.org/10.5114/pq.2020.96425
}

\author{
Shimaa H. Mohamed', Ghada E. Mohamed², Dalia M. Mosaad², Alshaymaa S. Abdelazeim², Ahmed F. Genedy³, \\ Haytham M. Elhafez ${ }^{2}$ \\ ${ }_{1}^{1}$ Physical Therapy Department, Alhawamdyia National Hospital, Giza, Egypt \\ ${ }^{2}$ Basic Science Department, Faculty of Physical Therapy, Cairo University, Giza, Egypt \\ ${ }^{3}$ Military Medical Academy and Agouza Rehabilitation Center, Giza, Egypt
}

Abstract

Introduction. The goal of this study was to compare the impact of ischaemic compression and kinesiotape on pain, physical function, and resting myoelectric activity of vastus medialis and vastus lateralis muscles in patients with unilateral acute patellar tendinitis.

Methods. Overall, 33 patients were randomly assigned to 3 equal groups. Group A received ischaemic compression and conventional treatment of patellar tendinitis. Group B received kinesiotape and conventional treatment of patellar tendinitis. Group $\mathrm{C}$ received only conventional treatment. Each patient received 3 sessions per week for 1 month. Pain intensity, physical function, and resting myoelectric activity were measured before, after, and after another 2 weeks of completion of the study.

Results. The results showed a statistically significant improvement in all the variables between the pre-intervention, post-intervention, and follow-up evaluations in the 3 groups $(p<0.05)$.

Conclusions. Kinesiotape is more effective than ischaemic compression in decreasing pain intensity level, improving physical function, and enhancing the activity of vastus lateralis and vastus medialis muscles in patients with unilateral acute patellar tendinitis.

Key words: kinesiotape, ischaemic compression, patellar tendinitis, resting myoelectric activity

\section{Introduction}

Patellar tendinitis is considered one of the most common conditions of overuse causing pain to the anterior knee area [1]. Its prevalence ranges from $31.9 \%$ to $44.6 \%$ [2]. It is commonly caused by repetitive jumping, cutting, or sprinting without rest between exercises, which severely loads the patellar tendon, as in basketball and volleyball players [1]. Ultimately, the tendon overload leads to tendon degeneration and, furthermore, reactive tendinopathy that weakens its structure and affects the career of subjects [3]. Witvrouw et al. [4] found that the patellar malalignment, patella alta, excessive laxity of the patellar tendon, muscle tightness, muscle imbalance, decreased flexibility of the quadriceps and hamstring muscles, and defects in knee extensor mechanisms, heavy training, and tougher training surfaces were suggested as potential risk factors for jumper's knee.

The knee extensor mechanism (the quadriceps muscles) linkage into the quadriceps tendon connects apex of the patella bone to the tibial tuberosity and functions to extend the tibiofemoral joint. The patella bone functions as a pulley, which shifts the quadriceps force direction, increases the quadriceps moment arm, and increases the quadriceps efficiency [5]. Also, the oblique fibres of the vastus medialis help balance the lateral pull exerted on the patella by the vastus lateralis muscle. So, impaired quadriceps muscle flexibility exacerbate tendon strain during joint movement, leading to tendon overload and the development of patellar tendinitis. Atrophy or inhibition of the vastus medialis oblique muscle lead to the development of patella alta or patellar tracking and patellar tendinitis [6].
Anomalous fascial tension in the form of myofascial trigger points (MTPs) in the thigh muscles leads to uncoordinated quadriceps contraction, which, in turn, causes patellar tendon pain. Therefore, in cases of patellar tendinitis, therapists should not focus their treatment only on the tendon itself but also on releasing MTPs and removing motor incoordination, which is in the muscular fascia of the thigh region [7]. MTPs release techniques include invasive and noninvasive therapies. The non-invasive therapies involve ice application, heat therapy, spray and stretch technique, electric stimulation, ultrasound therapy, and ischaemic compression [8].

Patellar tendinitis affects young to adult athletes with a peak age of 15-40 years [9]. The condition can be categorized into acute, sub-acute, and chronic, depending on the onset stage. The acute stage ends within 2 weeks; the subacute stage starts 2 weeks and ends 6 weeks after onset; and the chronic stage continues for more than 6 weeks [10].

Many physical therapy approaches have been used to improve symptoms of patellar tendinitis, such as application of manual therapy in the form of myofascial release of the knee extensor muscles, shockwave therapy, rest, anti-inflammatory medications, weight reduction, and kinesiotaping [2].

Ischaemic compression is performed by compressing the trigger points with tolerable pain of progressive and strong intensity. The compression causes blocking of blood flow in the area to be treated followed by its resurgence after releasing the pressure. The local blood flow supplies the affected area with energy and nourishment and enhances lymphatic drainage and removing the accumulated waste products, so relieving pain and fascial restrictions caused

Correspondence address: Shimaa H. Mohamed, Physical Therapy Department, Alhawamdyia National Hospital, Alhawamdyia, Giza,

Egypt, e-mail: dr.shimaahosny@hotmail.com

Received: 27.12 .2019

Accepted: 30.03 .2020

Citation: Mohamed SH, Mohamed GE, Mosaad DM, Abdelazeim AS, Genedy AF, Elhafez HM. Effect of ischaemic compression versus kinesiotape on patellar tendinitis. Physiother Quart. 2021;29(1):47-55; doi: https://doi.org/10.5114/pq.2020.96425. 
by MTPs [11]. The ischaemic compression technique normalizes the neuromuscular dysfunction present in muscles with MTPs, induced by an excessive release of acetylcholine from an autonomic modulation triggered by the release of chemical substances that results in increased myoelectric activity in the resting state [12].

Gelmini et al. [13] assessed the effect of ischaemic compression on the myoelectric activity of the upper trapezius muscle in patients with MTPs in this muscle. They concluded that the ischaemic compression could alter the myoelectric activity of muscles containing trigger points.

However, there is lack of research about its clinical effectiveness in the treatment of patellar tendinitis [14]. Therefore, this study was performed to investigate the effect of manual ischaemic compression on quadriceps trigger points in patients with patellar tendinitis. Also, the aim was to contrast the efficacy of kinesiotape and ischaemic compression in the refinement of pain and improving resting myoelectric activity in subjects with patellar tendinitis.

Currently, kinesiotaping plays a key role in treating patellar tendinitis as it helps to reduce pain and improve function and activity by (1) realignment of fascia; (2) lifting the fascia and soft tissue above skin to create a space above the area of pain and inflammation; (3) providing sensory stimulation to reduce or limit movement; and (4) eliminating exudates through the lymph ducts [15]. There are many reviews that support kinesiotaping role in decreasing pain [16-18], improving muscle power [19], and correcting muscle tone by stabilizing the weakened muscle [20]. Choi [18] emphasized its role in improving muscle performance via testing the effect of kinesiotape on muscle tone in patients with shoulder pain using Myotone Pro. He concluded that the physical therapy approach including kinesiotape, heat, and electrical stimulation was more beneficial than routine physical therapy in the treatment of shoulder pain. Muscle tone is usually defined as limb resistance to passive motion, where the resistance arises from passive and involuntary active forces [21].

\section{Subjects and methods}

\section{Study design}

A randomized controlled clinical trial was performed at the library of electromyography at the Faculty of Physical Therapy, Cairo University, Egypt, during the period of March 2017 to May 2019.

\section{Sample size calculation}

The $\mathrm{G}^{*}$ Power software (version 3.1.9.2) was used to estimate the sample size. The calculation was based on the F-test. The rate of type I error was set at $5 \%$ (alpha $<0.05)$, with an effect size of 0.68 of pain, which was obtained from the pilot study, performed among 5 subjects in each group. Type II error rate was at $95 \%$ power. The minimum sample size suitable for the research was 27 subjects in 3 groups.

\section{Subjects}

A total of 36 subjects diagnosed with acute unilateral patellar tendinitis were recruited from the outpatient clinic of the College of Physical Therapy at Cairo University, Egypt. During assessment for eligibility (Figure 1), 3 individuals were excluded because they had received treatment within the study. They received verbal and written explanation of the research purpose and procedures. The subjects who signed the consent form (to take part in the study for a total period of 6 weeks) were randomly assigned into 3 groups of equal numbers. The randomization was conducted with a random generator and permuted blocks of same size. Group A received ischaemic compression in addition to conventional treatment 3 times per week for 4 weeks. Group B received kinesiotape in addition to conventional treatment 3 times per week for 4 weeks. Group $\mathrm{C}$ received only conventional treatment for 4 weeks.

\section{Inclusion criteria}

The included subjects:

1. Were of both sexes [3].

2. Were aged $14-25$ years [9].

3. Complained of unilateral acute patellar tendinitis.

4. Suffered from pain localized to the inferior pole of the patella [22]; the pain was aggravated with sustained positions (squatting, prolonged sitting, running) and with stair climbing [9].

5. Had at least 2 active MTPs in the quadriceps muscle [23].

6 . Presented with a pain level of 3-4 in the numeric rating scale (NRS) [24].

7. Were characterized by a body mass index of $19-29 \mathrm{~kg} / \mathrm{m}^{2}$ [25].

\section{Exclusion criteria}

Subjects were excluded if they:

1. Had undergone knee surgery or had been diagnosed with rheumatoid arthritis or knee osteoarthritis [26].

2. Were diagnosed with patellofemoral pain syndrome [27].

3. Were obese [28] or pregnant [29].

\section{Outcome measurements}

The first measurement in the study was the level of pain intensity. It was evaluated with NRS. Physical function, another measured outcome, was established by using the Western Ontario and McMaster Universities Osteoarthritis Index (WOMAC). The last assessed outcome was the resting myoelectric activity, presented as root mean square (RMS). The assessments were performed 3 times: before the study, after the intervention, and after subsequent 2 weeks (follow-up).

\section{Pain intensity assessment}

NRS was used for measuring pain intensity level. The numbers were written from 0 to 10 in an ascending order. Each subject was asked to circle the number best describing their level of pain [30]. NRS has an outstanding test-retest reliability [31].

\section{Physical function}

The Arabic version of the WOMAC index was used to assess the physical function. It contains 24 questions, divided into the pain subscale (5 questions), the stiffness subscale (2 questions), and the physical function subscale (17 questions). The scale has 5 response levels for each item: none (0), mild (1), moderate (2), severe (3), and extreme (4).

Each participant was asked to answer the questions independently by choosing the number that described their case. The score was determined by adding the subscale 


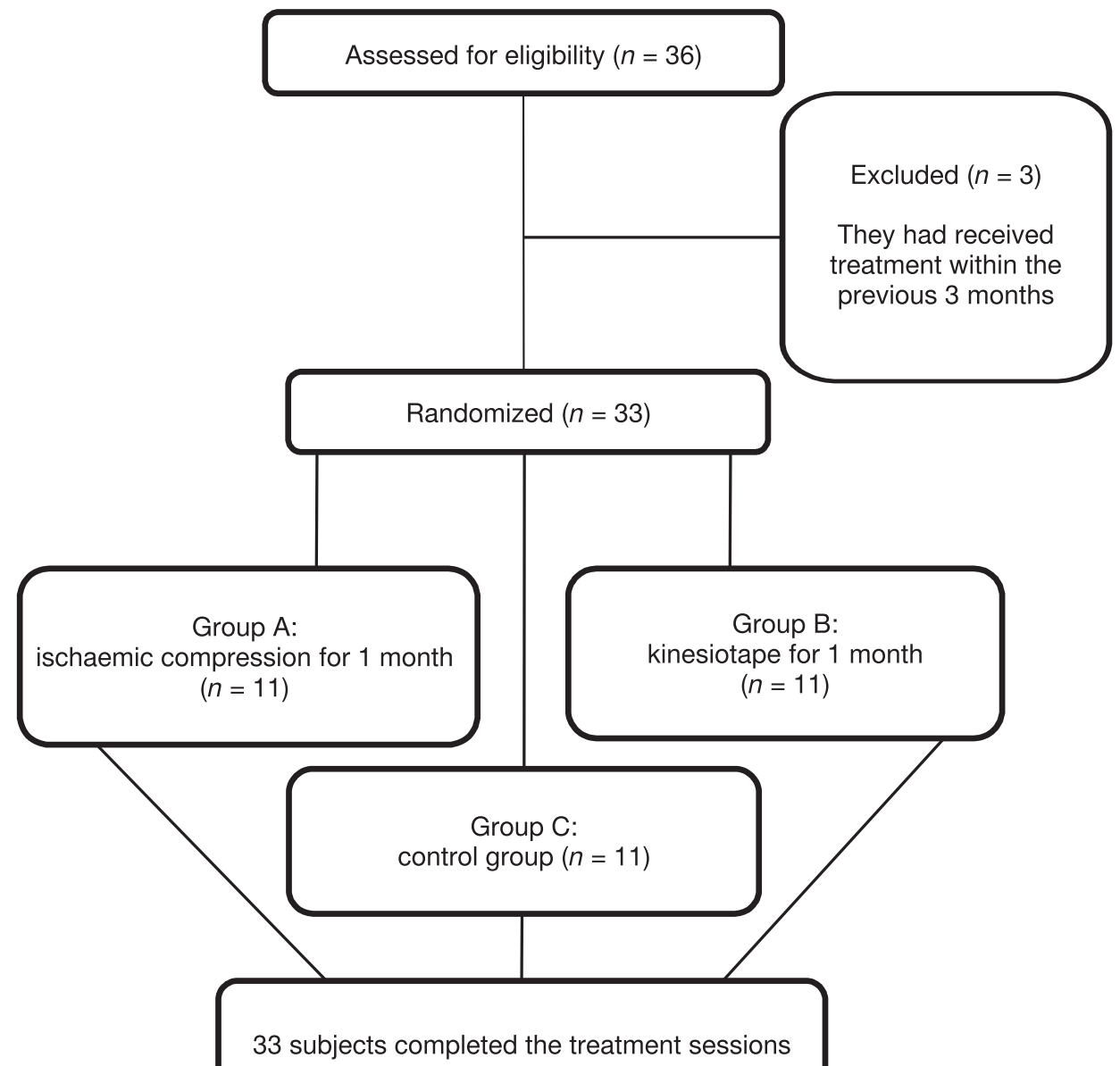

Figure 1. Study design

scores (pain: 0-20, stiffness: 0-8, physical function: 0-68). The final total scores collected (0-96) were determined by adding the scores for all subscales [32].

NRS can be used to qualify the disease prognosis or to check the utility of treatments (surgery, physiotherapy, etc.). The Arabic WOMAC index is a valid and reliable method for determining knee osteoarthritis severity, with metric properties in accordance [33].

\section{Resting myoelectric activity}

A 2-channel digital electromyography (EMG) device (Neuro-EMG-Micro, Neurosoft, Ivanovo, Russia) was used to assess the resting myoelectric activity of vastus medialis and vastus lateralis muscles. The subject was asked to sit in a relaxed position; then, the therapist cleaned the skin overlying the quadriceps and the knee joint with methylated alcohol to remove any elements that would increase skin resistance. The ground electrode was attached just above the tibial tuberosity and fixed by self-adhesive plastic. The vastus medialis and the vastus lateralis muscles were assessed separately [34].

For the vastus medialis muscle, 2 surface electrodes were placed $4 \mathrm{~cm}$ superior and $3 \mathrm{~cm}$ medial to the superomedial aspect of the patella in a longitudinal manner, with a 2-cm distance in between [35]. For the vastus lateralis muscle, 2 surface electrodes were placed at its mid belly (midpoint between the head of the greater trochanter and the lateral femoral epicondyle) [36] in a longitudinal manner, with a 2-cm distance in between [37].

\section{Testing protocol}

- At a resting position: the resting amplitude was recorded 3 times at a fully relaxed patient position; finally, the average was assumed.

- Normalization of the resting myoelectric activity: the therapist asked the patient to sit in a relaxed position (long sitting position). The subject was to perform an isometric contraction of knee extension with the knee in a fully extended position against resistance. Manual resistance was applied distal to the patella. Each contraction was sustained for 7 seconds and repeated 3 times, with a 30 -second rest in between [38].

- RMS calculation: the estimation of the normalized values was as follows: normalized RMS \% = EMG amplitude during resting / (average of EMGmax for the 3 trials) $\times 100$ [20]. The use of RMS circuit provides a nearly instantaneous output of the EMG power. This technique has somewhat a sounder mathematical basis than a simple linear envelope (integration). In the time domain, the RMS of the EMG signal is considered the most reliable parameter [39].

\section{Intervention}

Group A received ischaemic compression plus a conventional program for patellar tendinitis - in the form of strengthening and stretching exercises for knee flexors and extensors (Figure 2), and thigh adductors and abductors (Figure 3), as well as eccentric single-leg squats [40, 41]. Group B received kinesiotape plus a conventional program for patellar tendinitis. Group C (control group) received only a conven- 


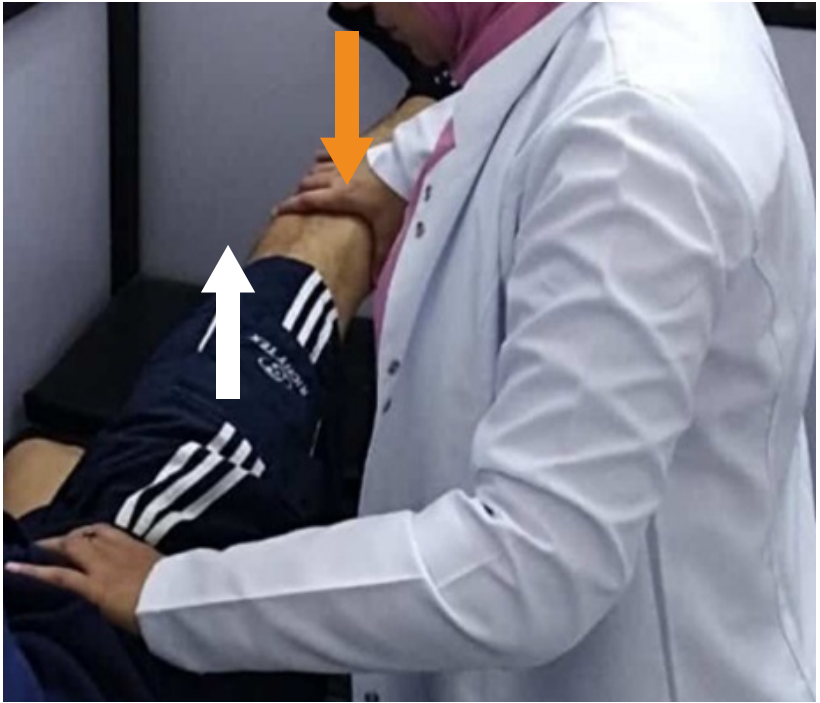

Figure 2. Strengthening of knee extensors

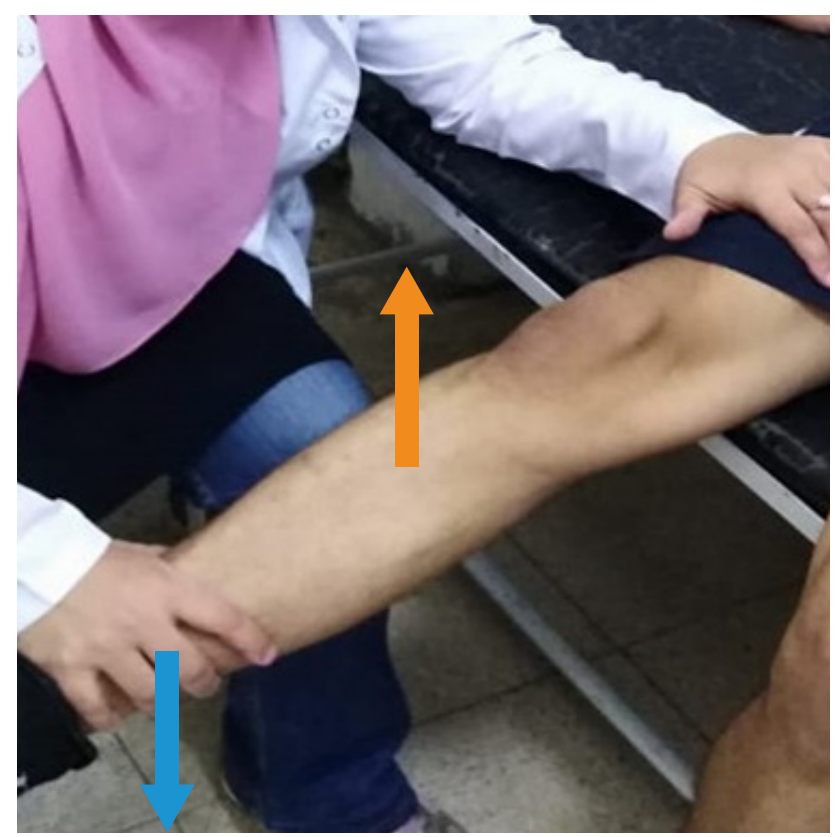

Figure 3. Strengthening of thigh abductors

tional program for patellar tendinitis.

\section{Ischaemic compression}

From a relaxed supine position, the subject was asked to determine the region of discomfort because they had active MTPs. The area was checked by pincer palpation and marked. Then, a therapist pressed the trigger point intermittently for 10-20 seconds, depending on the participant's tolerance. Next, the pressure gradually increased in accordance with the patient's tolerance and lasted for 90 seconds until the patient reported that the pain had lessened [42].

\section{Kinesiotape}

Group B received an adhesive tape that was waterproof and porous (Kinesio Tex, Kinesio USA, Albuquerque, USA). The kinesiotape was $5 \mathrm{~cm}$ in width and $0.5 \mathrm{~mm}$ in thickness [20].
During the kinesiotape application, the subject was rested in a supine position. Then, the taped region was uncovered, shaved, and cleaned with alcohol. The knee was flexed to a $90^{\circ}$ angle. The measurement of the tape began from midthigh to the tibial tuberosity. The tape was split at one end. It was stretched $10 \%$ amount of its original length and then applied vertically from mid-thigh down to the knee [43]. Each side of the split was applied around the patella [44].

\section{Conventional treatment}

The conventional program for patellar tendinitis $[40,41]$ included:

- Strengthening exercises for knee flexors and extensors, and thigh adductors and abductors. Each exercise was performed in 3 sets of 10 repetitions.

- Stretching exercises for knee flexors and extensors, and thigh adductors and abductors. Each stretch was maintained for 30 seconds and repeated 3 times, with a 1-minute rest in between.

- Eccentric single-leg squats with the use of a decline board with a $15-30^{\circ}$ decline and keeping the knee flexion less than $60^{\circ}$ in order not to excessively load the patellofemoral joint.

According to Dimitrios et al. [40], stretching exercises accompanied with eccentric squatting are effective in relieving pain and enhancing function in patients with patellar tendinitis.

\section{Statistical analysis}

The Shapiro-Wilk test was used to assess the normality of data distribution. All variables appeared normally distributed so the data were analysed by a parametric test. Mixed multilevel analysis of variance (MANOVA) served to determine the treatment and time effect at NRS, WOMAC, and normalized resting RMS of the vastus medialis and vastus lateralis muscles. The SPSS software, version 23 (IBM Corp, New York, USA) was applied, with an alpha level of $<0.05$.

\section{Ethical approval}

The research related to human use has complied with all the relevant national regulations and institutional policies, has followed the tenets of the Declaration of Helsinki, and has been approved by the research ethics committee of the Faculty of Physical Therapy, Cairo University (No. P.T. REC/01 21001513) and registered at the Pan African Clinical Trials Registry (Registry ID: PACTR-201702001948210).

\section{Informed consent}

Informed consent has been obtained from all individuals included in this study.

\section{Results}

\section{Demographic data}

All demographic data (age, weight, height, and BMI) were normally distributed, so analysis of variance (ANOVA) was used to compare the groups. There was no significant difference between the groups $(p>0.05)$, as shown in Table 1 .

\section{Outcomes results}

In general, there was a significant effect for treatment and time on all variables $(p<0.005, p<0.0001$, respectively). 
Table 1. Demographic data of the participants

\begin{tabular}{|c|c|c|c|c|c|c|}
\hline \multicolumn{2}{|c|}{ Characteristics } & Group A & Group B & Group C & $p$ & Significance \\
\hline \multirow{2}{*}{ Age (years) } & Mean & 20.23 & 24.54 & 22.36 & \multirow{2}{*}{0.08} & \multirow{2}{*}{ NS } \\
\hline & SD & 3.04 & 5.43 & 4.48 & & \\
\hline \multirow{2}{*}{ Weight (kg) } & Mean & 64.27 & 65.97 & 61 & \multirow{2}{*}{0.43} & \multirow{2}{*}{ NS } \\
\hline & SD & 8.59 & 11.24 & 7.59 & & \\
\hline \multirow{2}{*}{ Height (cm) } & Mean & 162.27 & 164.69 & 161.27 & \multirow{2}{*}{0.35} & \multirow{2}{*}{ NS } \\
\hline & SD & 7.69 & 5.15 & 4.65 & & \\
\hline \multirow{2}{*}{ BMI $\left(\mathrm{kg} / \mathrm{m}^{2}\right)$} & Mean & 24.41 & 24.11 & 23.34 & \multirow{2}{*}{0.65} & \multirow{2}{*}{ NS } \\
\hline & SD & 2.69 & 3.03 & 2.61 & & \\
\hline
\end{tabular}

NS - not significant, BMI - body mass index

\section{Effect of treatment on NRS (Figure 4)}

\section{Within-group analysis}

The results for group A showed a significant decrease in pain level after the treatment and at follow-up $(p<0.0001$; percent of change: $73 \%$ and $82.6 \%$, respectively). Also, for group $B$, there was a significant decrease after the treatment and at follow-up $(p<0.0001$; percent of change: $83 \%$ and $91 \%$, respectively). Finally, in group $C$, there was a significant decrease after the treatment and at follow-up $(p<$ 0.0001 ; percent of change: $67 \%$ and $73 \%$, respectively).

\section{Between-group analysis}

There was no statistically significant difference between the 3 groups before the treatment $(p>0.05)$. After the treatment, there was no significant difference between groups $A$ and $\mathrm{B}$ or groups $\mathrm{A}$ and $\mathrm{C}(p=0.16, p=0.87$, respectively). But, there was a significant difference between groups $B$ and $C(p<0.01)$. At follow-up, there was no significant difference between groups $A$ and $B$ or groups $A$ and $C(p=0.12$, $p=0.15$, respectively). But there was significant difference between groups B and C $(p<0.001)$.

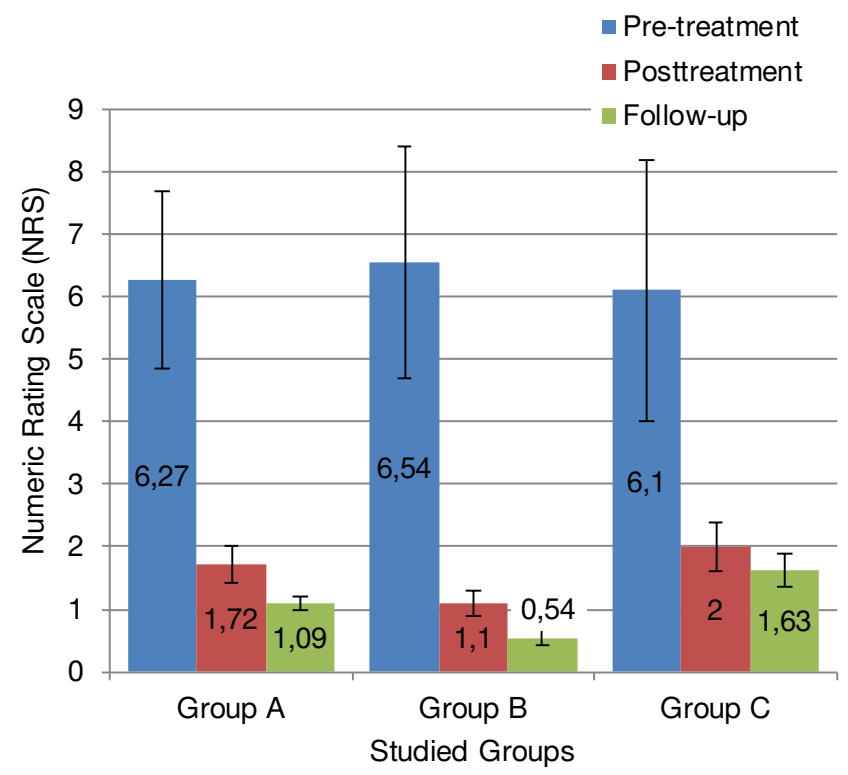

Figure 4. Mean and standard deviation of the numeric rating scale in the studied groups
Effect of treatment on vastus medialis RMS (Figure 5)

Within-group analysis

The results for group A showed no significant decrease in RMS after the treatment and at follow-up $(p>0.99, p>0.86$, respectively; percent of change: $3 \%$ and $13 \%$, respectively). But, for group B, there was a significant decrease after the treatment and at follow-up $(p<0.001, p<0.0001$, respectively; percent of change: $83 \%$ and $91 \%$, respectively). Finally, in group $C$, there was no significant decrease after the treatment $(p>0.104)$ but there was a significant decrease at follow-up ( $p<0.02$; percent of change: $67 \%$ and $73 \%$, respectively).

\section{Between-group analysis}

Before the treatment, there was no significant difference between groups $A, B$, and $C(p>0.05)$. After the treatment, there was no significant difference between groups $A$ and $B$, groups $A$ and $C$, or groups $B$ and $C(p=0.06, p=0.9, p=$ 0.31 , respectively). At follow-up, there was a significant difference between groups $A$ and $B$, and groups $B$ and $C(p=$ $0.004, p=0.03$, respectively). But there was no significant difference between groups $A$ and $C(p>0.9)$.

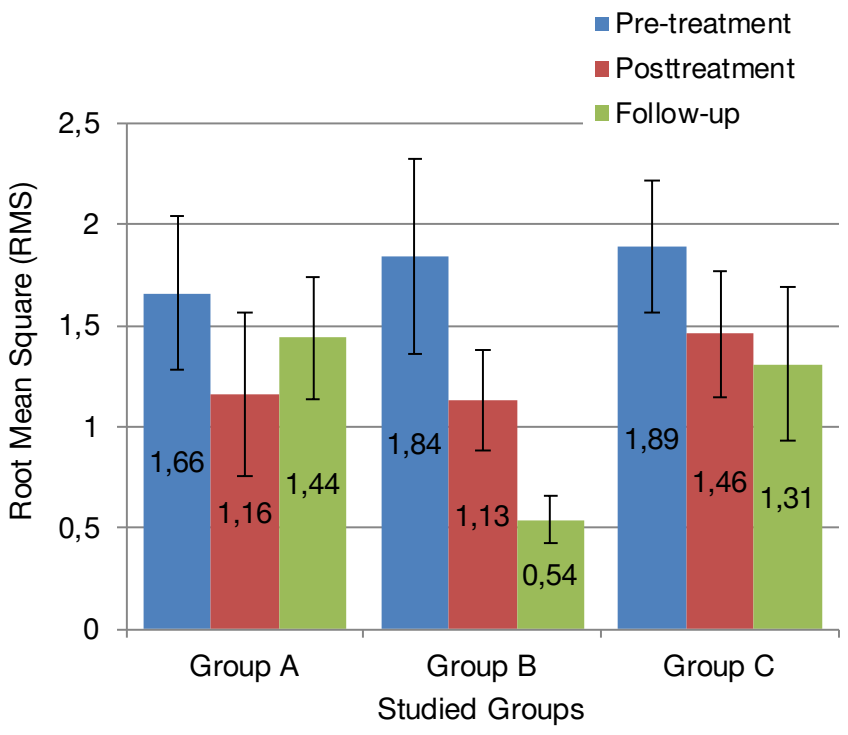

Figure 5. Mean and standard deviation of vastus medialis root mean square in the studied groups 


\section{Effect of treatment on vastus lateralis RMS (Figure 6)}

\section{Within-group analysis}

The results of group A showed a significant decrease in RMS after the treatment and at follow-up $(p<0.001, p<0.008$, respectively; percent of change: $29 \%$ and $32 \%$, respectively). Also, for group $B$, there was a significant decrease after the treatment and at follow-up $(p<0.0001, p<0.0001$, respectively; percent of change: $48 \%$ and $59 \%$, respectively). Finally, in group $\mathrm{C}$, there was no significant decrease after the treatment or at follow-up ( $p>0.44, p>0.31$, respectively; percent of change: $10.3 \%$ and $16 \%$, respectively).

\section{Between-group analysis}

Before the treatment, there was no significant difference between groups $A, B$, and $C(p>0.05)$. After the treatment, there was no significant difference between groups $A$ and B or groups $A$ and $C$ ( $p=0.13, p=0.17$, respectively). But,

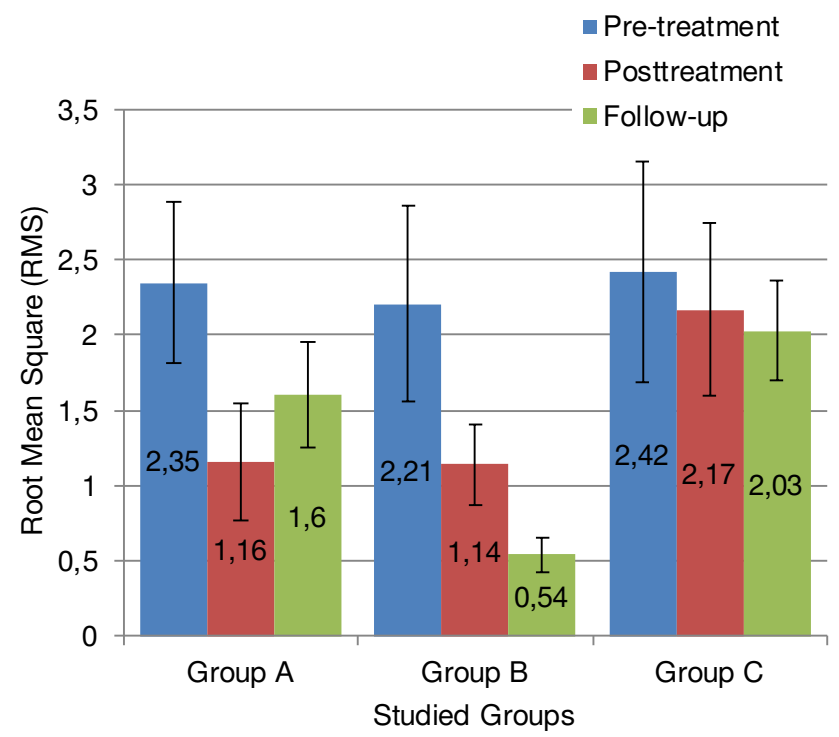

Figure 6. Mean and standard deviation of vastus lateralis root mean square in the studied groups

WOMAC - Western Ontario and McMaster Universities Osteoarthritis Index

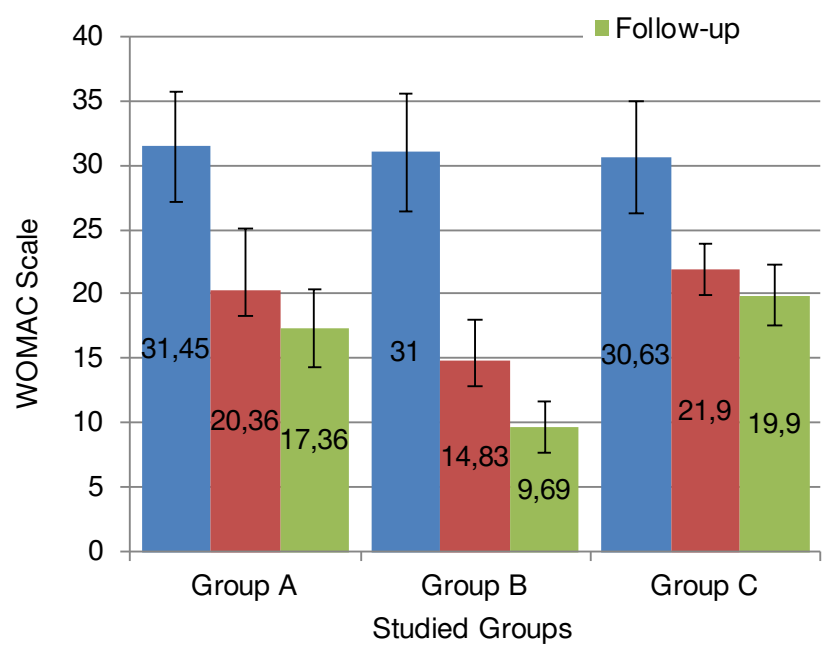

Figure 7. Mean and standard deviation of the WOMAC index in the studied groups there was a significant difference between groups $\mathrm{B}$ and $\mathrm{C}$ $(p<0.001)$. At follow-up, there was a significant difference between groups $A$ and $B$, and groups $B$ and $C(p<0.02, p<$ 0.0001 , respectively). But there was no significant difference between groups $A$ and $C(p>0.3)$.

\section{Effect of treatment on the WOMAC index (Figure 7)}

\section{Within-group analysis}

The results of group A showed a significant decrease of the WOMAC index after the treatment and at follow-up ( $p<$ 0.0001 ; percent of change: $35.5 \%$ and $45 \%$, respectively). Also, for group $B$, there was a significant change after the treatment and at follow-up $(p<0.0001$; percent of change: $53 \%$ and $68 \%$, respectively). Finally, in group C, there was a significant decrease after the treatment and at follow-up ( $p<0.0001$; percent of change: $28 \%$ and $35 \%$, respectively).

\section{Between-group analysis}

Before the treatment, there was no significant difference between groups $\mathrm{A}, \mathrm{B}$, and $\mathrm{C}(p>0.05)$. After the treatment, there was a significant difference between groups $A$ and $B$ and groups $B$ and $C(p=0.002, p=0.001$, respectively). But there was no significant difference between groups $A$ and $C(p=0.98)$. At follow-up, there was a significant difference between groups $A$ and $B$ and groups $B$ and $C(p=0.001$, $p=0.001$, respectively). But there was no significant difference between groups $A$ and $C(p=0.142)$.

\section{Discussion}

The current study was designed to compare the effects of kinesiotape and ischaemic compression on the rehabilitation of acute patellar tendinitis. The group that received kinesiotape showed more refinement than the ischaemic compression group and the control group. Kinesiotape has a key role in inhibiting myofascial pain by lifting the skin, which leads to increased circulation, enhanced lymphatic drainage (helping remove oedema by directing the exudates towards a lymph duct), and decreased pressure on nociceptors [20]. Kinesiotape application stimulates ligament/tendon correction: an increased stimulus over a ligament/tendon area results in an increased stimulus of mechanoreceptors to be perceived as proprioceptive stimulus that stimulates more normal tissue [45].

According to Kumbrink [46], the tape enhances tonus regulation as it causes change in muscle tonus via activation of skin receptors which strengthen additional peripheral afferent signals (joint, muscle, skin). The change in the resting myoelectric activity in the kinesiotape group may be due to the application form. The application of kinesiotape from insertion to origin results in an eccentric pull on the underlying fascia that inhibits muscle activity [20].

The results of the present study are in agreement with those obtained by Ptaszkowski et al. [47], who studied the effect of muscle energy techniques vs. kinesiotape on resting bioelectric activity and visual analogue scale in upper trapezius pain. The results showed a reduction in muscle activity and visual analogue scale in the kinesiotape group. Similarly, the current study is matched with the results achieved by Massei et al. [44], who tested the effectiveness of therapeutic taping (kinesiotape and leukotape) on pain, range of motion, power, balance, and strength in individuals with and without acute patellar tendinopathy - and stated that kine- 
siotape and leukotape had an effect on some variables in certain individuals.

In the same line, Cho [48] et al. investigated the effect of kinesiotaping on pain, proprioception, and range of motion in older patients with knee osteoarthritis and demonstrated that kinesiotape attenuated various types of pain and improved range of motion and proprioception in osteoarthritis patients.

Also, the results of the present study showed that ischaemic compression was an effective manual therapy technique for MTP release as the ischaemia following long pressure allows a circulatory influence, enhances blood circulation, relieves transient blood flow occlusion, produces a reactive hyperaemia, and helps get rid of waste products. These wastes stimulate pain receptors, causing continuous pain. So, hyperaemia following ischaemic compression leads to pain relief [49]. This idea is supported by Gazbare et al. [50], who compared the effects of ultrasound therapy and ischaemic compression in the treatment of trigger points and found that ischaemic compression should be a preferred therapy for MTPs in a physical therapy setup as it was effective, easily available, accessible, cost effective, and nondependent on any machines; moreover, the results can be seen in a short period.

This study validates the idea that pain in the front of the knee may be initiated by trigger points in the quadriceps muscle causing muscle weakness. It is supported by Elsayed et al. [14], who stated that the presence of trigger points in the quadriceps muscle was considered one of the most important causes of anterior knee pain, and manual ischaemic compression had been reported to be effective in treating trigger points. Also, the present results agree with a study by Ravichandran et al. [42], who compared ischaemic compression and ultrasound therapy of MTPs in the trapezius muscle. They used a visual analogue scale to test the pain threshold before and after application. The results confirmed that the ischaemic compression technique led to better improvement in the functional outcome in neck pain.

The current study revealed that kinesiotaping decreased RMS. This result is supported by a study performed by Abd El-Azeim et al. [20], who tested the effect of kinesiotape on the normalized bioelectric activity of trapezius trigger points and concluded that the method was effective in decreasing the muscle activity in the resting state.

Aguilar-Ferrándiz et al. [17] investigated the effect of kinesiotape on the gastrocnemius muscle activity. They recorded the muscle activity by means of a Kine-Pro Motion Wireless surface EMG for a total of 7 seconds and rectified and normalized by the EMG value of the maximum voluntary isometric contraction. The study reported that kinesiotape could enhance muscle activity.

The current study revealed that ischaemic compression might improve the electromagnetic activity of the quadriceps muscle after releasing the trigger points. These results are supported by those achieved by Gelmini et al. [13], who assessed the effect of ischaemic compression on the myoelectric activity of the upper trapezius muscle in patients with MTPs in this muscle and concluded that the ischaemic compression could alter the myoelectric activity of muscles containing trigger points.

\section{Limitations}

The time of treatment was 4 weeks only, which is considered relatively short.

\section{Conclusions}

Kinesiotape is more effective than ischaemic compression in decreasing pain intensity, improving physical function, and enhancing activity of vastus lateralis and vastus medialis muscles in patients with unilateral acute patellar tendinitis.

\section{Acknowledgments}

The authors express their gratitude to the subjects who participated in this study.

\section{Disclosure statement}

No author has any financial interest or received any financial benefit from this research.

\section{Conflict of interest}

The authors state no conflict of interest.

\section{Funding}

This research did not receive any specific grant from funding agencies in the public, commercial, or not-for-profit sectors.

\section{References}

1. Larsson B, Karlsson S, Eriksson M, Gerdle B. Test-retest reliability of EMG and peak torque during repetitive maximum concentric knee extensions. J Electromyogr Kinesiol. 2003;13(3):281-287; doi: 10.1016/s10506411(03)00022-1.

2. Rees JD, Stride M, Scott A. Tendons - time to revisit inflammation. Br J Sports Med. 2014;48(21):1553-1557; doi: 10.1136/bjsports-2012-091957.

3. Rudavsky A, Cook J. Physiotherapy management of patellar tendinopathy (jumper's knee). J Physiother. 2014; 60(3):122-129; doi: 10.1016/j.jphys.2014.06.022.

4. Witvrouw E, Bellemans J, Lysens R, Danneels L, Cambier D. Intrinsic risk factors for the development of patellar tendinitis in an athletic population. A two-year prospective study. Am J Sports Med. 2001;29(2):190-195; doi: 10.1177/03635465010290021201.

5. Dan M, Parr W, Broe D, Cross M, Walsh WR. Biomechanics of the knee extensor mechanism and its relationship to patella tendinopathy: a review. J Orthop Res. 2018;36(12):3105-3112; doi 10.1002/jor.24120.

6. Neumann DA. Kinesiology of the musculoskeletal system. Foundations for rehabilitation, $2^{\text {nd }}$ ed. St. Louis: Mosby; 2001.

7. Pedrelli A, Stecco C, Day JA. Treating patellar tendinopathy with fascial manipulation. J Bodyw Mov Ther. 2009; 13(1):73-80; doi: 10.1016/j.jbmt.2008.06.002.

8. De Paula VRM, de Paula GM, Justi J, Soares CS, Sanchez HM. Comparative study between ischemic compression and dry needling in myofascial pain syndrome: possibilities in health. Man Ther Posturology Rehabil J. 2019;23:623; doi:10.17784/mtprehabjournal.2018.16.623.

9. Volpi P, Prospero E, Bait C, Carimati G, Di Francia VP, Felisaz P, et al. Patellar tendinopathy. In: Bisciotti GM, Volpi $P$ (eds.), The lower limb tendinopathies: etiology, biology and treatment. Cham: Springer; 2016; 165-179.

10. Rosso F, Bonasia DE, Cottino U, Dettoni F, Bruzzone M, Rossi R. Patellar tendon: from tendinopathy to rupture. Asia Pac J Sports Med Arthrosc Rehabil Technol. 2015; 2(4):99-107; doi: 10.1016/j.asmart.2015.07.001.

11. Kashyap R, Iqbal $A$, Alghadir $\mathrm{AH}$. Controlled intervention to compare the efficacies of manual pressure release and the muscle energy technique for treating mechanical 
neck pain due to upper trapezius trigger points. J Pain Res. 2018;11:3151-3160; doi: 10.2147/JPR.S172711.

12. Bigongiari A, Franciulli PM, Souza FdA, Mochizuki L, Araujo RC. Surface electromyography activity analysis of the myofascial trigger points [in Portuguese]. Rev Bras Reumatol. 2008;48(6):319-324; doi: 10.1590/S048250042008000600003.

13. Gelmini TÂM, Sant'Anna PCF, Dhein W, La Torre M. Acute effect of the ischemic compression technique on the EMG activity of the muscle upper trapezius in subjects with myofascial triggers points. Man Ther Posturology Rehabil J. 2018;16:583; doi: 10.17784/mtprehabjournal.2018.16.583.

14. Elsayed SEBA, Ammar TARA, Tolba AMN, Elgeidi AARE. Manual ischemic compression in patients with patellofemoral pain syndrome. The $18^{\text {th }}$ International Scientific Conference Faculty of Physical Therapy, Cairo, Egypt; 16-17 March, 2017. 2017; doi: 10.13140/RG.2.2.23562. 77761.

15. Lyman KJ, Keister K, Gange K, Mellinger CD, Hanson TA. Investigating the effectiveness of Kinesio ${ }^{\circledR}$ taping space correction method in healthy adults on patellofemoral joint and subcutaneous space. Int J Sports Phys Ther. 2017;12(2):250-257.

16. Abd El-Khalik MM, Abd El-Monem MG, Mohammed AH, Abd El-Fattah MA. Effect of exercises and Kinesio tape on range of motion of neck and jaw post partial glossectomy. Med J Cairo Univ. 2017;85(1):47-56.

17. Aguilar-Ferrándiz ME, Castro-Sánchez AM, MataránPeñarrocha GA, García-Muro F, Serge T, Moreno-Lorenzo C. Effects of Kinesio taping on venous symptoms, bioelectrical activity of the gastrocnemius muscle, range of ankle motion, and quality of life in postmenopausal women with chronic venous insufficiency: a randomized controlled trial. Arch Phys Med Rehabil. 2013;94(12): 2315-2328; doi: 10.1016/j.apmr.2013.05.016.

18. Choi J-H. Effects of Kinesio taping on muscle tone, stiffness in patients with shoulder pain. J Korean Soc Phys Med.2017;12(3):43-47; doi:10.13066/kspm.2017.12.3.43.

19. Ahn IK, Kim YL, Bae Y-H, Lee SM. Immediate effects of kinesiology taping of quadriceps on motor performance after muscle fatigued induction. Evid Based Complement Alternat Med. 2015;2015:410526; doi: 10.1155/2015/ 410526.

20. Abd El-Azeim AS, Elhafez HM, Ahmed SEB, Draz AH, Kattabei OM. Efficacy of Kinesio tape on pressure pain threshold and normalized resting myoelectric activity on upper trapezius myofascial trigger points (a randomized clinical trial). J Adv Pharm Edu Res. 2019;9(3):413-418.

21. Gurfinkel V, Cacciatore TW, Cordo P, Horak F, Nutt J, Skoss R. Postural muscle tone in the body axis of healthy humans. J Neurophysiol. 2006;96(5):2678-2687; doi: 10.1152/jn.00406.2006.

22. Malliaras P, Cook JL, Purdam C, Rio E. Patellar tendinopathy: clinical diagnosis, load management, and advice for challenging case presentations. J Orthop Sports Phys Ther. 2015;45(11):887-898; doi: 10.2519/jospt. 2015.5987.

23. Perez-Palomares S, Oliván-Blázquez B, Arnal-Burró AM, Mayoral-Del Moral O, Gaspar-Calvo E, de-la-Torre-Beldarraín ML, et al. Contributions of myofascial pain in diagnosis and treatment of shoulder pain. A randomized control trial. BMC Musculoskelet Disord. 2009;10:92; doi: 10.1186/1471-2474-10-92.

24. Biernat R, Trzaskoma Z, Trzaskoma L, Czaprowski D. Rehabilitation protocol for patellar tendinopathy applied among 16- to 19-year old volleyball players. J Strength Cond Res. 2014;28(1):43-52; doi: 10.1519/JSC.0b013 e31829797b4.

25. Taș S, Yılmaz S, Onur MR, Soylu AR, Altuntaş O, Korkusuz F. Patellar tendon mechanical properties change with gender, body mass index and quadriceps femoris muscle strength. Acta Orthop Traumatol Turc. 2017;51(1): 54-59; doi: 10.1016/j.aott.2016.12.003.

26. Morgan S, Coetzee FF. Proposing a patellar tendinopathy screening tool following a systematic review. S Afr J Physiother. 2018;74(1):454; doi: 10.4102/sajp.v74i1.454.

27. Lopes AD, Hespanhol Jr LC, Kamper SJ, Costa LOP. Exercise for patellar tendinopathy. Cochrane Database Syst Rev. 2018;2018(7):CD013078; doi: 10.1002/1465 1858.CD013078.

28. Fairley J, Toppi J, Cicuttini FM, Wluka AE, Giles GG, Cook J, et al. Association between obesity and magnetic resonance imaging defined patellar tendinopathy in community-based adults: a cross-sectional study. BMC Musculoskelet Disord. 2014;15:266; doi: 10.1186/14712474-15-266.

29. Tang X, Gao Z, Li Y, Yu J, Li X. Efficacy and safety of focused ultrasound versus microwave therapy for cervical ectopy: a meta-analysis. J Evid Based Med. 2015;8(2): 61-74; doi: 10.1111/jebm.12150.

30. Snijders GF, van den Ende CHM, van den Bemt BJF, van Riel PLCM, van den Hoogen FHJ, den Broeder AA, et al. Treatment outcomes of a Numeric Rating Scale (NRS)-guided pharmacological pain management strategy in symptomatic knee and hip osteoarthritis in daily clinical practice. Clin Exp Rheumatol. 2012;30(2):164170.

31. Alghadir AH, Anwer S, lqbal A, lqbal ZA. Test-retest reliability, validity, and minimum detectable change of visual analog, numerical rating, and verbal rating scales for measurement of osteoarthritic knee pain. J Pain Res. 2012;11:851-856; doi: 10.2147/JPR.S158847.

32. Faik A, Benbouazza $K$, Amine B, Maaroufi $H$, Bahiri $R$, Lazrak N, et al. Translation and validation of Moroccan Western Ontario and McMaster Universities (WOMAC) osteoarthritis index in knee osteoarthritis. Rheumatol Int. 2008;28(7):677-683; doi: 10.1007/s00296-007-0498-z.

33. Sweelam AM, Abd El-Mageed SF, Gomaa EF, Azzam AH. Effect of pronated foot posture on the Arabic version of WOMAC index in women with medial compartment knee osteoarthritis. Med J Cairo Univ. 2018;86(3):1111-1117; doi: 10.21608/mjcu.2018.56125.

34. Wong Y-M, Ng GYF. Surface electrode placement affects the EMG recordings of the quadriceps muscles. Phys Ther Sport. 2006;7(3):122:127; doi: 10.1016/j.ptsp. 2006.03.006.

35. Hubbard JK, Opersteny S. EMG analysis of two portions of the vastus medialis muscle during selected knee rehabilitation exercises. J Musculoskelet Res. 2002;6(2): 107-118; doi: 10.1142/S0218957702000812.

36. Kushion D, Rheaume J, Kopchitz K, Glass S, Alderink G, Jinn J-H. EMG activation of the vastus medialis oblique and vastus lateralis during four rehabilitative exercises. Open Rehabil J. 2012;5(1):1-7; doi: 10.2174/18749437 01205010001.

37. Al Amer HS, Sabbahi MA, Alrowayeh HN, Bryan WJ, Olson SL. Electromyographic activity of quadriceps muscle during sit-to-stand in patients with unilateral knee osteoarthritis. BMC Res Notes. 2018;11(1):356; doi: 10.1186/s13104-018-3464-9.

38. McLean $L$. The effect of postural correction on muscle activation amplitudes recorded from the cervicobrachial 
region. J Electromyogr Kinesiol. 2005;15(6):527-535; doi: 10.1016/j.jelekin.2005.06.003.

39. Fukuda TY, Echeimberg JO, Pompeu JE, Lucareli PRG, Garbelotti S, Gimenes RO, et al. Root mean square value of the electromyographic signal in the isometric torque of the quadriceps, hamstrings and brachial biceps muscles in female subjects. J Appl Res. 2010;10(1):32-39.

40. Dimitrios S, Pantelis M, Kalliopi S. Comparing the effects of eccentric training with eccentric training and static stretching exercises in the treatment of patellar tendinopathy. A controlled clinical trial. Clin Rehabil. 2012;26(5): 423-430; doi: 10.1177/0269215511411114.

41. Rio E, Purdam C, Girdwood M, Cook J. Isometric exercise to reduce pain in patellar tendinopathy in-season: is it effective "on the road"? Clin J Sport Med. 2019;29(3): 188-192; doi: 10.1097/JSM.0000000000000549.

42. Ravichandran P, Ponni HK, Aseer PAL. Effectiveness of ischemic compression on trapezius myofascial trigger points in neck pain. Int J Physiother. 2016;3(2):186-192; doi: 10.15621/ijphy/2016/v3i2/94883.

43. Elhafez HM. Kinesiotaping techniques. Personal communication, Professor of physical therapy, Basic Science Department, Faculty of Physical Therapy, Cairo University; 2017.

44. Massei M, Przysucha E, Sanzo P. The effects of therapeutic taping on pain, range of motion, power, balance, and strength, in athletes and non-athletes with and without acute patellar tendinopathy. Int J Health Sci. 2015; 3(4):9-20; doi: 10.15640/ijhs.v3n4a2.

45. Kase K, Tatsuyuki H, Tomoko O. Development of kinesiotape. In: Kinesio Taping Association (eds.), Kinesio taping perfect manual. Albuquerque: Kinesio; 1996; 117-118.

46. Kumbrink B. The K-taping method. In: Kumbrink B (ed.), K-taping, $2^{\text {nd }}$ ed. Dortmund: Springer Medizin; 2016; 1-11.

47. Ptaszkowski K, Slupska L, Paprocka-Borowicz M, KołczTrzęsicka A, Zwierzchowski K, Halska U, et al. Comparison of the short-term outcomes after postisometric muscle relaxation or Kinesio taping application for normalization of the upper trapezius muscle tone and the pain relief: a preliminary study. Evid Based Complement Alternat Med. 2015;2015:721938; doi: 10.1155/2015/ 721938.

48. Cho H-Y, Kim E-H, Kim J, Yoon YW. Kinesio taping improves pain, range of motion, and proprioception in older patients with knee osteoarthritis: a randomized controlled trial. Am J Phys Med Rehabil. 2015;94(3):192200; doi: 10.1097/PHM.0000000000000148.

49. Esparza D, Aladro-Gonzalvo AR, Rybarczyk Y. Effects of local ischemic compression on upper limb latent myofascial trigger points: a study of subjective pain and linear motor performance. Rehabil Res Pract. 2019;2019: 5360924; doi: 10.1155/2019/5360924.

50. Gazbare P, Palekar TJ, Rathi M. Comparison of ultrasound and ischemic compression on latent trigger point in upper trapezius. Int J Pharm Bio Sci. 2014;5(4):B635B642. 